\title{
How long do population level field experiments need to be? A meta-analysis across the 40-year old LTER network
}

\author{
Sarah Cusser ${ }^{1}$, Jackson Helms IV ${ }^{1}$, Christie Bahlai ${ }^{2}$, and Nick Haddad ${ }^{2}$ \\ ${ }^{1}$ Michigan State University \\ ${ }^{2}$ Michigan State University W K Kellogg Biological Station
}

November 12, 2020

\begin{abstract}
Long-term experiments are important in evaluating ecosystem properties and processes that are slow to develop or require proper evaluation over an appropriately variable climate. We repurpose the wealth of data accessible through the forty-year-old Long-Term Ecological Research (LTER) network with a novel moving window algorithm and meta-analysis approach to ask if aspects of study taxa or environment alter the extent of research necessary to detect consistent results, or the proportion of spurious short-term trends. We found that experimental studies focused on plants, and those conducted in dynamic abiotic environments, were characterized by longer critical temporal thresholds and more spurious trends. Further, nearly half of the studies we investigated required 10 years or longer to reach a temporal threshold, and 4 studies (of 100) required longer than 20 years. We champion long-term data and argue that long-term experiments are more necessary than ever to understand, explain, and predict long-term trends.
\end{abstract}

\section{Hosted file}

Cusser_et_al._MS_EL_10_NOV_2020.pdf available at https://authorea.com/users/375303/articles/ 492586-how-long-do-population-level-field-experiments-need-to-be-a-meta-analysis-acrossthe-40-year-old-lter-network 